\title{
Renormalization of Derivative Coupling Theories
}

\author{
R. Arnowitt and S. Deser* \\ The Institute For Advanced Study, Princeton, New Jersey
}

(Received June 24, 1955)

\begin{abstract}
The method of functional integrals is applied to the problem of meson theories with derivative couplings. In the static limit, solutions in closed form can be exhibited. The infinities occurring in the theory are found to be removable in terms of $Z_{2}$ and mass renormalizations, contrary to the conclusions of perturbation analysis. The divergences occurring here have the form of essential singularities, in contradistinction to the branch-point behavior of the usual "renormalizable" theories. The lack of validity of the perturbation expansion is thereby accounted for. These techniques can be extended to treat the full recoil neutral $p s(p v)$ problem omitting closed loops. The theory is represented in terms of an exponential coupling which permits a nonperturbation series solution for the various propagators. Two infinite renormalizations are again required. The resultant functions are given mean-
\end{abstract}

ing by analytic continuation procedures which are adapted to the four-dimensional nature of the problem. The form of the effective coupling suggests a rearrangement of the answer in terms of exponentials of the meson propagator. As a result mass operator-like structures can be defined. These explicitly exhibit the transcendental nature of the coupling and generalized equivalence theorems with $p s(p s)$ theory can be generated. In a similar fashion, effective interaction operators for the two-nucleon and mesonnucleon Green's functions are derived. The possible applicability of these quantities to questions of physical interest such as nuclear potentials and multiple meson production is briefly mentioned.

In an appendix, a model of beta coupling is discussed in connection with the renormalization question there.

\section{INTRODUCTION}

$\mathrm{F}^{\circ}$ OLLOWING the success of the renormalization program in electrodynamics, the same methods and concepts have been brought to bear on the various types of meson theories. The success in this region, however, has not been universal. The applicability of perturbation renormalization techniques has become a criterion dividing these theories into two classes.

The most salient characteristic of many of the "unrenormalizable" theories is that they involve gradients of the meson field in the interaction. Upon examination of the radiative corrections occurring in these cases, one discovers that the infinities appearing in successive orders become more violent, due to the linear (or higher) dependence upon momentum transfer of the elementary vertex. Further, the infinities appear in such a way that an infinite number of observables would have to be renormalized. Such effects would correspond, in terms of a cut-off picture, to a theory entirely dependent upon the details of the structure of the elementary particles. ${ }^{1}$ These derivative interaction theories, whose coupling constants have dimensions of reciprocal length, were thought to require, along with other so-called theories of the second kind, the existence of a fundamental length to give them meaning. ${ }^{2}$ The possibility has always remained open, however, that the series of divergences might, in reality, sum to a more tractable form.

The meson theories of most current interest are, of course, the pseudoscalar ones. Although the pseudoscalar theory with pseudovector coupling has long seemed to be physically attractive, it has been neglected

\footnotetext{
* F. B. Jewett Fellow. Now at the Institute for Theoretical Physics, Copenhagen, Denmark.

${ }^{1}$ Sakata, Umezawa, and Kamefuchi, Progr. Theoret. Phys. 7, 377 (1952).

2 W. Heisenberg, Ann. Physik 32, 20 (1938).
}

in recent years because of the above troubles. It is the purpose of the earlier sections of the paper to treat the problems involved in some simple nonrecoil models of derivative coupling theories. Already in these examples will be found many of the basic difficulties and divergences which beset the complete $p s(p v)$ theory.

By means of the functional integral formulation of field theory, one can obtain closed-form solutions in these cases. In this procedure (which will be the basic tool employed throughout) the complete, radiatively corrected, nucleon Green function, for example, is obtained by considering the propagation of a bare particle in an arbitrary external field distribution averaged over all such possible fields. This process represents the emission and subsequent absorption of the virtual meson field. The problem is thereby divided into two separate parts: that of finding a first quantized nucleon propagator in an arbitrary external field and that of averaging with the appropriate weight factor over this field (the functional integration). Perturbation theory corresponds to a Born approximation solution of the first problem. For the initial cases to be considered, the static, nonrecoil limit will be taken, which is equivalent to having the first quantized kernel satisfy a one dimensional equation. The latter can be solved rigorously and the functional integrations performed for the structures obtained. The steps then necessary to give these theories meaning will be clarified, as will the concept of renormalization. It will be seen in precisely what respects the perturbation analysis of renormalization fails; in addition, the infinities to be deleted from the theory will be exhibited explicitly.

Another set of unrenormalizable theories, not directly related to the above, consists of the so-called direct interactions, exemplified by the Fermi couplings. In Appendix A, a simple model (in that the heavy particles, though not the leptons, are taken as static) of 
scalar beta coupling is considered and the problems of renormalization discussed in the light of our methods.

In the meson case, the chief problem of physical interest must take fully into account all the degrees of freedom of the nucleon field, both the particle and spin motion. To treat the full recoil theory for neutral mesons, omitting closed loop diagrams, the method of functional integration is again the basic technique employed. The procedure used in this problem is to note that the ostensibly linear pseudovector coupling is equivalent to a pseudoscalar coupling exponential in the boson field. This fact allows the functional integrations to be performed and the infinite renormalizations separated. In this representation, the solutions are themselves exponential in the coupling constant. Thus structures similar to those obtained in the simpler models appear again in the complete problem, though in a more complicated context.

Another similarity to the problems noted in the nonrecoil cases is the necessity for giving definition to the propagators by means of analytic continuation. The apparatus developed here extends the treatment required for the case of branch points first considered in the simple renormalizable theory to the more singular derivative coupling four dimensional problems. There is actually a qualitative difference between the role played by the continuation procedure in these two cases due to the more violent nature of the essential singularities. This is true even for the null $s(v)$ theory with recoil, treated in Appendix D.

The expansion appropriate for performing the renormalizations is perhaps not that most suited to display the physical content of the theory. Since the effective coupling structure is transcendental, a natural series in such problems to obtain physical results would seem to involve exponentials in the coupling constant rather than merely the coupling constant itself. One finds many generalized equivalence theorems between the $p s(p s)$ and $p s(p v)$ theories in this process. By this analysis, the consequencies of the theory can be couched in diagrammatic language which displays how the natural exponential structure in the meson propagator replaces the perturbation form. Thus one can speak of an effective mass operator upon which can perhaps be based a reasonable approximation scheme. The above procedure is, of course, applicable to the more complex Green functions such as the meson-nucleon and twonucleon ones. In both cases interaction operators can be defined; in view of some of the attractive properties of $p s(p v)$ theory, particularly at low energies and (in this formulation) with regard to multiple production, these quantities are briefly investigated.

\section{FUNCTIONAL INTEGRAL FORMULATION}

Throughout this work we shall employ the functional formulation of field theory to discuss the quantities of interest. ${ }^{34}$ We consider a Lagrangian of the form ${ }^{5}$

$$
\begin{aligned}
& \mathscr{L}(x)=-\frac{1}{2} \bar{\psi}(x)(\gamma p+m) \psi(x)+\text { herm conj } \\
& \quad-\frac{1}{2} \phi(x)\left(k^{2}+\mu^{2}\right) \phi(x)-g \bar{\psi}(x) \Gamma f[\phi(x)] \psi(x),
\end{aligned}
$$

where $\Gamma$ represents the fundamental vertex $(\Gamma \phi=\phi$ for neutral scalar theory, $\gamma_{5} \tau^{i} \phi^{i}$ for $p s(p s)$ symmetric theory, $i \gamma_{5} \gamma_{\mu} \partial_{\mu} \phi$ for neutral $p s(p v)$ theory, etc.) and $f(\phi)$ equal $\phi(x)$ for a linear coupling, $\phi^{2}(x)$ for a pair theory, etc. The one-nucleon propagator with all radiative corrections (but omitting vacuum polarization) may be obtained from the functional integral

$$
\begin{gathered}
G_{+}^{\prime}\left(x, x^{\prime}\right)=N^{-1} \int \exp \left[-\frac{1}{2} i \int \phi(\xi) \Delta_{+}^{-1}\left(\xi, \xi^{\prime}\right) \phi\left(\xi^{\prime}\right) d \xi d \xi^{\prime}\right] \\
\times G_{+}\left(x, x^{\prime} ; \phi\right) \delta \phi ; \\
N=\int \exp \left[-\frac{1}{2} i \int \phi \Delta_{+}{ }^{-1} \phi d \xi d \xi^{\prime}\right] \delta \phi
\end{gathered}
$$

where $\phi(\xi)$ is a $c$-number over which the functional integration is carried out, $\Delta_{+}$obeys the equation $\left(-\partial_{\mu}^{2}+\mu^{2}\right) \Delta_{+}\left(\xi, \xi^{\prime}\right)=\delta\left(\xi-\xi^{\prime}\right)$ and $G_{+}(\phi)$ is the bare Green's function in the external field $\phi$, satisfying

$$
\{\gamma p+m+g \Gamma f[\phi(x)]\} G_{+}\left(x, x^{\prime} ; \phi\right)=\delta\left(x-x^{\prime}\right) .
$$

The "+" subscript indicates the usual outgoing wave boundary conditions. We also shall have occasion to use the free particle Green's function $G_{+}{ }^{(0)}\left(x-x^{\prime}\right)$ $=\left(x\left|(\gamma p+m)^{-1}\right| x^{\prime}\right)$.

Similarly, the general Green's function governing the propagation of $n$ nucleons and $m$ mesons is given by

$$
\begin{aligned}
G_{n m}{ }^{\prime}\left(x_{1} \cdots x_{n}, x_{1}{ }^{\prime} \cdots x_{n}{ }^{\prime} ; \xi_{1} \cdots \xi_{m}\right) \\
=N^{-1} i^{[m / 2]} \int \exp \left[-\frac{1}{2} i \int \phi \Delta_{+}{ }^{-1} \phi d \xi d \xi^{\prime}\right] \\
\quad \times G_{+}\left(x_{1} x_{1}{ }^{\prime}\right) \cdots G_{+}\left(x_{n}, x_{n}{ }^{\prime}\right) \phi\left(\xi_{1}\right) \cdots \phi\left(\xi_{m}\right) \delta \phi
\end{aligned}
$$

where $[m / 2]$ means "integral part of $m / 2$ " and the nucleon coordinates are to be suitably antisymmetrized.

\section{DERIVATIVE COUPLING THEORIES IN THE NONRECOIL LIMIT}

Although our chief interest will center around the renormalization difficulties appearing in the derivative coupling theories, we first indicate how even so simple an example as neutral scalar theory without recoil exhibits one kind of problem we shall encounter in further work. For this case, in the Dirac Lagrangian, $\gamma p+m$ is replaced by $-p_{0}+m .{ }^{6}$ In this approximation

${ }^{3}$ S. F. Edwards and R. E. Peierls, Proc. Roy. Soc. (London) A224, 24 (1954).

${ }^{4}$ S. Deser, Phys. Rev. 99, 325 (1955).

${ }^{5}$ Our $\gamma_{\mu}$ are defined by the relation $\left\{\gamma_{\mu}, \gamma_{\nu}\right\}=-2 \delta_{\mu \nu}$, and $\gamma_{5}^{2}=-1$

${ }^{6}$ This treatment of the nucleons is equivalent to the usual fixed source approximation. 
the solution to $G_{+}{ }^{\prime}\left(t-t^{\prime}\right)$ is given in reference 3 ; the relevant point here is that the renormalized Green function behaves at small times as

$$
\begin{array}{cc}
G_{c} \sim \exp \left[-\left(g^{2} / \pi^{2}\right) \ln \mu\left(t-t^{\prime}\right)\right] & t>t^{\prime} \\
0 & t<t^{\prime} .
\end{array}
$$

The existence of a Fourier transform of the convergent propagator is necessary in order to maintain the quantum mechanical transformation theory for finite times. Further, in momentum space, the Green function must behave appropriately to meet the usual physical requirements on the theory. It is clear, from Eq. (5), that a critical coupling strength occurs at $g^{2} / 4 \pi=\pi / 4$, above which rigorously no transform exists. Since physically such a limitation on the size of $g^{2}$ would appear to have no meaning, we proceed to remove it by one of the various mathematical techniques invented for this purpose. ${ }^{7}$ In particular, the method that we will adopt here and elsewhere will be that of an analytic continuation of $g^{2} / \pi^{2}$ from below the critical value of unity to above it. Specifically we consider the function $G_{c}\left(t-t^{\prime}, g^{2}\right)$ for values of $g^{2} / \pi^{2}<1$. Here we may evaluate its transform $G_{c}\left(p_{0}, g^{2}\right)$ which is analytic in $g^{2}$ for $g^{2} / \pi^{2}<1$. Such a function will have a continuation to values above unity (though poles will occur when $\left.g^{2} / \pi^{2}=1,2,3, \cdots\right)$. Thus meaning has been given for $G_{c}$ beyond the barrier that the particular representation in Eq. (5) seemed to impose. Insofar as the resultant behavior in momentum space is concerned, the results of reference 3 should hold for the larger coupling constants.

We now consider a set of examples which both involve derivatives of the meson field in the coupling term and are simple enough to be solved exactly. These will arise from various approximations to be made on the nucleon's motion as represented in Eq. (3) in the case where the coupling is pseudovector. Such approximations do not involve the exponential weight factor in the functional integration which represents the radiative corrections due to the boson field. We begin with a somewhat artificial limit in which both the nucleon recoil and the gradient parts of the coupling have been dropped:

$$
\left(-p_{0}+m-i g \gamma_{5} \partial_{0} \phi\right) G_{+}\left(t, t^{\prime} ; \phi\right)=\delta\left(t-t^{\prime}\right),
$$

where $G_{+}\left(t, t^{\prime}\right)$ actually includes a $\delta\left(r-r^{\prime}\right)$. The $\gamma_{5}$ has been retained in Eq. (6) in order to include one feature of $\gamma_{5}$ couplings: $\gamma_{5}^{2}=-1$. (Terms linear in $\gamma_{5}$ will, of course, turn out to vanish in the solution since negative energy states have been otherwise neglected.)

Equation (6) may easily be integrated to yield

$$
\begin{aligned}
G_{+}\left(t, t^{\prime} ; \phi\right)=i \theta\left(t-t^{\prime}\right) e^{-i m\left(t-t^{\prime}\right)} & \\
& \times \exp \left[-g \gamma_{5} \int_{t^{\prime}}^{t} \partial_{0} \phi(\tau) d \tau\right],
\end{aligned}
$$

${ }^{7}$ F. J. Dyson has pointed out in conversation that methods of L. Schwartz may also be applied to this problem. where $\theta(x)$ is 1 or 0 as $x$ is greater or less than zero (which enforces the nonrelativistic boundary conditions). The functional integration may be performed via the formula

$$
\begin{aligned}
N^{-1} \int \exp \left[-\frac{1}{2} i \int \phi(\xi) \Delta_{+}{ }^{-1}\left(\xi, \xi^{\prime}\right) \phi\left(\xi^{\prime}\right) d \xi d \xi^{\prime}\right. \\
\left.+\int \phi(\xi) f(\xi) d \xi\right] \\
=\exp \left[-\frac{1}{2} i \int f(\xi) \Delta_{+}\left(\xi, \xi^{\prime}\right) f\left(\xi^{\prime}\right) d \xi d \xi^{\prime}\right] .
\end{aligned}
$$

In Eq. (7), $f(\xi)=-g \gamma_{5} \partial_{0} H\left(\xi_{0} ; t, t^{\prime}\right) \delta^{3}(\xi)$, where $H\left(\xi_{0}\right.$; $\left.t, t^{\prime}\right)=\theta\left(t-\xi_{0}\right) \theta\left(\xi_{0}-t^{\prime}\right)$. The presence of $\gamma_{5}$ in the exponential is not troublesome since it commutes with all other quantities involved. The total Green function is then

$$
\begin{aligned}
G_{+}{ }^{\prime}\left(t-t^{\prime}\right)=i \theta\left(t-t^{\prime}\right) e^{-i m\left(t-t^{\prime}\right)} & \\
& \times \exp \left\{-i g^{2}\left[\Delta_{+}\left(t-t^{\prime}\right)-\Delta_{+}(0)\right]\right\} .
\end{aligned}
$$

All that is necessary to renormalize the theory, therefore, is to identify $\exp i g^{2} \Delta_{+}(0)$ with $Z_{2}$, no mass renormalization being required. ${ }^{8}$ The form $\Delta_{+}\left(t-t^{\prime}\right)$ $-\Delta_{+}(0)$ appearing in Eq. (9) arises since

$$
\operatorname{disc} G_{+}{ }^{\prime}(0)=i\langle\{\bar{\psi}(t), \psi(t)\}\rangle=i \text {. }
$$

The renormalization just performed is qualitatively different from those needed in a "renormalizable" theory such as the neutral scalar one quoted above. In particular, the infinity appears as an essential singularity rather than as a branch point (the latter manifesting itself in perturbation theory as a series of logarithms). If Eq. (6) and hence $G_{+}{ }^{\prime}$ were solved in a perturbation fashion, one would have found the usual "unrenormalizable" results: The higher and higher diagrams would have higher and higher infinities in them, leading one to believe that an infinite number of renormalizations were necessary. Actually, as can be seen in Eq. (9), the infinities sum to an essential singularity which, however, is a harmless multiplicative factor. Indeed, if $G_{+}{ }^{\prime}(t)$ is only a function of $\Delta_{+}(t)$ $-\Delta_{+}(0)$, the condition that theory be renormalizable in the usual way,

$$
G_{+}^{\prime}\left[\Delta_{+}(t)-\Delta_{+}(0)\right]=Z_{2}\left[\Delta_{+}(0)\right] G_{c}\left[\Delta_{+}(t)\right]
$$

forces an exponential solution like Eq. (9) and hence an essential singularity.

The renormalized one-particle Green function in its dependence on time is everywhere finite and does not even exhibit the difficulties in Fourier analysis discussed for the neutral scalar theory. The higher Green's functions [which may be obtained from Eq. (4)], such

${ }^{8} \mathrm{It}$ is interesting to note that this theory formally has a Ward identity. This appears to be the case whenever there are no commutation problems for a nonrecoil meson theory. 
as the two-nucleon propagator, may all be renormalized in an analogous fashion. However, in both theories analytic continuation must be applied to Fourier analyze these more complex functions. In the neutral scalar case it is again a matter of branch points while here an essential singularity on the light cone must be treated. The requisite technique for such problems will be introduced later.

As can be seen by inspecting the various Green functions, the theory we have been considering here produces no observable effects in the $S$-matrix. In this it resembles the situation encountered in $s(v)$ coupling. Indeed the latter's nonrelativistic $\Gamma \phi$ is $\partial_{0} \phi$. Similar statements can be made about the renormalization and lack of physical effects for theories of the type $\Gamma \phi$ $=\partial_{0}{ }^{n} \phi .^{9}$ While all such examples make no physical predictions, they do retain enough of the dynamical structure to illustrate the type of renormalization and the analytic continuation problems that will arise in the physically interesting cases. In Appendix B, a static approximation involving gradient coupling and having observable consequences is discussed.

\section{THE NONRECOIL APPROXIMATION AND TRANSCENDENTAL COUPLING}

The first approximation scheme considered, Eq. (6), neglected anticommutativity of $\gamma_{5}$ with $\gamma_{0}$ while including the fact that $\gamma_{5}^{2}=-1$. A scheme that embodies part of this effect starts from the equation

$$
\left(-\gamma_{0} p_{0}+m+i g \gamma_{5} \gamma_{0} \partial_{0} \phi\right) G_{+}\left(t, t^{\prime} ; \phi\right)=\delta\left(t-t^{\prime}\right) .
$$

We now make a transformation analogous to that introduced by Dyson ${ }^{10}$ :

$$
\begin{aligned}
G_{+}\left(t, t^{\prime} ; \phi\right)=\exp \left[-g \gamma_{5} \phi(t)\right] H_{+}\left(t, t^{\prime} ; \phi\right) & \\
& \times \exp \left[-g \gamma_{5} \phi\left(t^{\prime}\right)\right] .
\end{aligned}
$$

The equation obeyed by $H_{+}$is

$\left\{-\gamma_{0} p_{0}+m \exp \left[-2 g \gamma_{5} \phi(t)\right]\right\} H_{+}\left(t, t^{\prime} ; \phi\right)=\delta\left(t-t^{\prime}\right)$.

Having used the fact that $\left\{\gamma_{0}, \gamma_{5}\right\}=0$, which is essentia 1 in arriving at Eq. (14), we now make the approximation of setting $\gamma_{0}=1 .^{11}$ As will be seen later, this step changes considerably the predictions of the theory which therefore differs from the true nonrecoil limit of $p s(p v)$. The approximation is simple enough, however, to enable us to justify expansions that will later be generalized to more complicated cases.

Within this framework, then, Eq. (14) may be

${ }^{9}$ That nonrecoil theories of the type $\Gamma \phi=\partial_{0}{ }^{n} \phi$ are renormalizable has also been discovered by L. Cooper, following paper [Phys. Rev. 100, 362 (1955)].

${ }_{10}$ F. J. Dyson, Phys. Rev. 73, 929 (1948).

11 An approximation substantially equivalent to this has been discussed by R. J. Glauber, Phys. Rev. 84, 395 (1951). In that paper the fact that the scheme was renormalizable was observed and the $S$-matrix obtained. Our Eq. (16) differs from Glauber's result by the inclusion of the first two exponentials and the existence of $Z_{2}$. In the transition from the finite times Green's function to the renormalized $S$-matrix, these terms disappear. integrated to yield

$$
H_{+}\left(t, t^{\prime} ; \phi\right)=i \theta\left(t-t^{\prime}\right) \exp \left[-i m \int_{t^{\prime}}^{t} e^{-2 g \gamma_{5} \phi(\tau)} d \tau\right] \text {. }
$$

In order to perform the functional integral it is necessary to expand $H_{+}$in a power series in $m$. This expansion, which should be valid at small times, will be justified later. The resulting exponentials may be functionally integrated term by term to yield

$$
\begin{aligned}
& G_{+}{ }^{\prime}\left(t-t^{\prime}\right)=Z_{2} i \theta\left(t-t^{\prime}\right) \\
& \times \exp \left[i g^{2} \Delta_{+}\left(t t^{\prime}\right)\right] \sum_{n=0}^{\infty} \frac{\left(-i m^{\prime}\right)^{n}}{n !} \int_{t^{\prime}}^{t} d t_{1} \cdots d t_{n} \\
& \times \exp \left[2 i g^{2} \sum_{i=1}^{n}\left\{\Delta_{+}\left(t_{i} t\right)+\Delta_{+}\left(t_{i} t^{\prime}\right)\right\}\right] \\
& \times \exp \left[4 i g^{2} \sum_{i>j=1}^{n} \Delta_{+}\left(t_{i}, t_{j}\right)\right],
\end{aligned}
$$

where $Z_{2}$ and the renormalized mass $m^{\prime}$ are given by

$$
\begin{aligned}
& Z_{2}=\exp \left[i g^{2} \Delta_{+}(0)\right], \\
& m^{\prime}=m \exp \left[2 i g^{2} \Delta_{+}(0)\right] .
\end{aligned}
$$

It is interesting that $Z_{2}$ here is formally greater than unity if, in the spirit of a static theory, one sets $\mathbf{r}$ to zero in $\Delta_{+}(0)$ before $t$. The fact that all signs in the exponentials are positive is explicitly due to the dropping of $\gamma_{0}$ in Eq. (14) and also entails that disc $G_{+}{ }^{\prime}(0)$ in Eq. (16) does not reduce to $i$. Thus this approximation destroys the canonical commutation relations.

Although the infinities obtained here are similar to those arising in previous approximations where the functional integral could be done rigorously, it may be asked whether this theory is indeed infinite or whether the difficulties actually come from the small time expansion and the subsequent functional integration of the series. To consider this question, we first obtain an expression for $G_{+}\left(t, t^{\prime} ; \phi\right)$ which is rigorously correct at small times. In general one has

$$
\begin{aligned}
& G_{+}\left(t, t^{\prime} ; \phi\right)=i \theta\left(t-t^{\prime}\right) \exp \left[-g \gamma_{5}\left\{\phi(t)+\phi\left(t^{\prime}\right)\right\}\right] \\
& \quad \times \exp \left[-i m \int_{t}^{t} d \tau\left\{\cos 2 g \phi(\tau)-\gamma_{5} \sin 2 g \phi(\tau)\right\}\right] .
\end{aligned}
$$

We may replace the integrals occurring in Eq. (18) by the integrands evaluated at some mean value since the $\phi$ 's are real functions. Denoting below by $t_{1}$ and $t_{2}$ the mean value points for the cosine and sine integrals respectively, Eq. (18) may be written

$$
\begin{aligned}
G_{+}\left(t, t^{\prime} ; \phi\right)=i \theta\left(t-t^{\prime}\right) \cos \left[g\left\{\phi(t)+\phi\left(t^{\prime}\right)\right\}\right. \\
\left.-i m\left(t-t^{\prime}\right) \sin 2 g \phi\left(t_{2}\right)\right] \\
\times \exp \left[-i m\left(t-t^{\prime}\right) \cos 2 g \phi\left(t_{1}\right)\right],
\end{aligned}
$$

where terms odd in $\phi$ have been dropped, since they vanish in the subsequent integration. The functional 
integral, $\int \delta \phi$, may be viewed as an infinite product of ordinary integrals, $\Pi_{i} \int d \phi\left(x_{i}\right)$, over all cells in space. The range of each integration is from $-\infty$ to $+\infty$. As $\phi\left(t_{1}\right)$ and $\phi\left(t_{2}\right)$ cover this range, $\left(t-t^{\prime}\right) \cos 2 g \phi\left(t_{1}\right)$ and $\left(t-t^{\prime}\right) \sin 2 g \phi\left(t_{2}\right)$ remain bounded and tend to zero as $t \rightarrow t^{\prime}$. Thus in the entire range of functional integration the usual power series in $t-t^{\prime}$ is uniformly convergent. Hence if the functional integral of (19) exists, then the integral of the power series in $t-t^{\prime}$ exists. To first order one may write

$$
\begin{aligned}
G_{+}\left(t, t^{\prime} ; \phi\right) \cong i \theta\left(t-t^{\prime}\right)\left[\cos g\left\{\phi(t)+\phi\left(t^{\prime}\right)\right\}\right. \\
+i m\left(t-t^{\prime}\right)\left\{\sin 2 g \phi\left(t_{2}\right) \sin g\left\{\phi(t)+\phi\left(t^{\prime}\right)\right\}\right. \\
\left.-\cos 2 g \phi\left(t_{1}\right) \cos g\left\{\phi(t)+\phi\left(t^{\prime}\right)\right\}\right],
\end{aligned}
$$

which gives upon functional integration

$$
\begin{aligned}
G_{+}{ }^{\prime}\left(t-t^{\prime}\right) \cong & Z_{2} i \theta\left(t-t^{\prime}\right) \exp \left[i g^{2} \Delta_{+}\left(t-t^{\prime}\right)\right]\left[1-i m^{\prime}\left(t-t^{\prime}\right)\right. \\
& \times\left\{\cos 2 g^{2}\left(\Delta_{+}\left(t_{1}-t\right)+\Delta_{+}\left(t_{1}-t^{\prime}\right)\right)\right. \\
& \left.\quad+i \sin 2 g^{2}\left(\Delta_{+}\left(t_{2}-t\right)+\Delta_{+}\left(t_{2}-t^{\prime}\right)\right\}\right] .
\end{aligned}
$$

This is equal, of course, to the first two terms in the expansion (16) when the mean value condition is used at sufficiently small times. The important point is that the series expansion of Eq. (19) when integrated has the same infinities as Eq. (16), and thus the infinite renormalizations are not due to the process of expansion but are intrinsic properties of the theory.

\section{SOLUTION AND RENORMALIZATION OF THE THEORY WITH RECOIL}

The radiatively corrected one-nucleon propagator for the full recoil neutral $p s(p v)$ theory is given by the same formal functional integral as in the no-recoil cases, Eq. (2). Now, however, $G_{+}\left(x x^{\prime} ; \phi\right)$ obeys the more complicated equation

$$
\left[\gamma p+m+i g \gamma_{5} \gamma_{\mu} \partial_{\mu} \phi\right] G_{+}\left(x x^{\prime} ; \phi\right)=\delta\left(x-x^{\prime}\right) .
$$

One can again bring this into the form in which the transcendental aspect of the coupling is emphasized by the same substitution as was made in Sec. 4:

$$
\begin{aligned}
G_{+}\left(x x^{\prime} ; \phi\right)=\exp [ & \left.-g \gamma_{5} \phi(x)\right] \\
& \times H_{+}\left(x x^{\prime} ; \phi\right) \exp \left[-g \gamma_{5} \phi\left(x^{\prime}\right)\right] .
\end{aligned}
$$

$H_{+}\left(x x^{\prime} ; \phi\right)$ now obeys the equation

$$
\left\{\gamma p+m \exp \left[-2 g \gamma_{5} \phi(x)\right]\right\} H_{+}\left(x x^{\prime} ; \phi\right)=\delta\left(x-x^{\prime}\right) \text {. }
$$

A closed form solution here would require the disentangling of even more complicated ordered operator expressions than in the nonrelativistic case. However, the analysis there given of the convergence of a series development of the corresponding equation in powers of the mass times the propagation time, $t-t^{\prime}$, would seem to sanction a similar expansion in this case. We therefore obtain the operator equation

$$
H_{+}=\frac{1}{\gamma p} \sum_{n=0}^{\infty}\left(\exp \left[-2 g \gamma_{5} \phi\right]^{-m}\right)^{n} \text {. }
$$

$m / \gamma p$ thus plays the role of $-m / p_{0}$ in the no-recoil case; hence one is making an expansion in large momenta or small space-time intervals. Defining the neutrino-like free propagator, $H_{0}\left(x x^{\prime}\right)=\left(x\left|(\gamma p)^{-1}\right| x^{\prime}\right)$, Eq. (25) becomes in the coordinate representation

$$
\begin{aligned}
H_{+}\left(x x^{\prime} ; \phi\right)=\sum_{n=0}^{\infty}( & -m)^{n} \int \cdots \int d y_{1} \cdots d y_{n} H_{0}\left(x y_{1}\right) \\
& \quad \times \exp \left[-2 g \gamma_{5} \phi\left(y_{1}\right)\right] H_{0}\left(y_{1} y_{2}\right) \\
& \quad \times \exp \left[-2 g \gamma_{5} \phi\left(y_{2}\right)\right] \cdots H_{0}\left(y_{n} x^{\prime}\right) .
\end{aligned}
$$

With the exponentials in the positions above, the functional integration cannot be explicitly performed. Now, however, that the operator form of Eq. (25) has been expressed in coordinate functions, the exponentials can be freely commuted past the $H_{0}$ 's to the extreme right by noting that

$$
\begin{aligned}
\exp \left[-2 g \gamma_{5} \phi(y)\right] H_{0}\left(y^{\prime} y^{\prime \prime}\right) & \\
& =H_{0}\left(y^{\prime} y^{\prime \prime}\right) \exp \left[2 g \gamma_{5} \phi(y)\right] .
\end{aligned}
$$

One obtains then for $G_{+}\left(x x^{\prime} ; \phi\right)$ the structure

$$
\begin{gathered}
G_{+}\left(x x^{\prime} ; \phi\right)=\sum_{n=0}^{\infty}(-m)^{n} \int d y_{1} \cdots d y_{n} H_{0}\left(x y_{1}\right) \cdots H_{0}\left(y_{n} x^{\prime}\right) \\
\times \exp \left\{-2 g \gamma_{5} \int d \xi \phi(\xi)\left[\frac{\delta\left(\xi-x^{\prime}\right)}{2}+\frac{(-)^{n+1} \delta(\xi-x)}{2}\right.\right. \\
\left.\left.-\delta\left(\xi-y_{n}\right)+\delta\left(\xi-y_{n-1}\right)+\cdots+(-)^{n} \delta\left(\xi-y_{1}\right)\right]\right\} . \quad(28)
\end{gathered}
$$

The usefulness of the neutrino Green's function expansion is now clear; had one attempted to expand Eq. (24) in a series of $G_{+}{ }^{(0)}$ 's by artifically adding and subtracting $m$, one would have encountered considerably more difficulty in moving the exponentials to one side. A more important problem than this combinatorial one, however, would have been the extraction of the infinite mass renormalization which will turn out to be trivial for the form of Eq. (28). After the renormalization we shall indeed proceed to restate (in Sec. 7) the answer in terms of propagators with masses. Equation (28) more clearly exhibits the fact that we are here dealing with an expansion about the origin in coordinate space; the first term is just $H_{0}\left(x x^{\prime}\right)$ which has the same leading singularity at small times as the rigorous $G_{+}\left(x x^{\prime} ; \phi\right) . .^{12}$

The functional integral can now be performed to

12 These singularities are, of course, just those of $G_{+}{ }^{(0)}\left(x, x^{\prime}\right)$. The infrared divergences which exist in $\mathrm{Eq}$. (28) due to the fact that we have formally expanded a problem with recoil about $m=0$, will, of course, disappear as the series is recombined into propagators with mass. 
yield for the one-nucleon Green function

$$
\begin{aligned}
& G_{+}{ }^{\prime}\left(x x^{\prime}\right)=Z_{2} \sum_{n=0}^{\infty}\left(-m^{\prime}\right)^{n} \exp \left[-i g^{2}(-)^{n} \Delta_{+}\left(x x^{\prime}\right)\right] \\
& \times \int d y_{1} \cdots d y_{n} H_{0}\left(x y_{1}\right) \cdots H_{0}\left(y_{n} x^{\prime}\right) \\
& \times \exp \left\{2 i g^{2} \sum_{i=1}^{n}(-)^{i}\left[\Delta_{+}\left(x y_{i}\right)+(-)^{n+1} \Delta_{+}\left(y_{i} x^{\prime}\right)\right]\right\} \\
& \times \exp \left\{4 i g^{2} \sum_{i>j=1}^{n}(-)^{i+j} \Delta_{+}\left(y_{i} y_{j}\right)\right\},
\end{aligned}
$$

where

$$
Z_{2}=\exp \left[i g^{2} \Delta_{+}(0)\right] ; \quad m^{\prime}=m \exp \left[2 i g^{2} \Delta_{+}(0)\right] .
$$

It is shown in Appendix C that the result (29) is formally equivalent to the perturbation one.

The sign of the leading singularity at $x_{\mu}=0$ of $\Delta_{+}(0)$ is ambiguous, depending on whether the limit is taken in a space- or time-like fashion. In order to determine whether $Z_{2}$ is to be considered greater or less than unity one must insist on the preservation of the boundary condition that

$$
\operatorname{disc} G_{+}{ }^{\prime}\left(\mathbf{r}-\mathbf{r}^{\prime}, 0\right)=i\left\langle\left\{\bar{\psi}(\mathbf{r}), \psi\left(\mathbf{r}^{\prime}\right)\right\}\right\rangle=i \gamma_{0} \delta\left(\mathbf{r}-\mathbf{r}^{\prime}\right) .
$$

That this condition is built into $G_{+}{ }^{\prime}$ can most easily be seen from Eq. (24). For any reasonably behaved $\phi(x)$, $\gamma_{0} p_{0}$ is responsible for producing the singularity of Eq. (31) and, since the functional integral of Eq. (2) leaves this leading term unaffected, the desired result is guaranteed. In Eq. (29) the limiting procedure is not so transparent. However, as will become apparent after the process of analytic continuation has rendered the exponentials more meaningful near their singularities, the term linear in $H_{0}$ will still be chiefly responsible for producing the characteristic discontinuity. Since this term has for coefficient $\exp \left[-i g^{2}\left\{\Delta_{+}\left(x-x^{\prime}\right)-\Delta_{+}(0)\right\}\right]$ which must approach unity as $x_{\mu} \rightarrow x_{\mu}{ }^{\prime}$ in the space-like sense of Eq. (31), the sign of $\Delta_{+}(0)$ is thus determined to be the "space-like" one. That is, $Z_{2}$ for this theory has the physically desirable property of being $<1$ in magnitude.

\section{ANALYTIC CONTINUATION PROCESS}

While the renormalizations performed in Eq. (29) extract the appropriate infinite parts of the theory, the intermediate integrals as well as the Fourier analysis of the propagator must be given meaning. In this, the theory is akin to the simplest example of the static neutral scalar theory discussed in Sec. 3. Here, however, the procedures required are complicated by both the four-dimensional nature of the problem and the essential singularities in the functions being considered.

The formal Fourier transform of $G_{c}\left(x-x^{\prime}\right)$, assuming for the moment that all functions are well-behaved, may be written as

$$
\begin{aligned}
G_{c}(p)=\sum_{n=0}^{\infty}\left(-m^{\prime}\right)^{n} \int \cdots \int H_{0}\left(p-\sum_{i=1}^{n+1} k_{i, 0}\right) \cdots \\
\times H_{0}\left(p-\sum_{i=0}^{n-1} k_{n+1, i}-\sum_{i=0}^{n-1} k_{n, i}\right) \\
\times H_{0}\left(p-\sum_{i=0}^{n} k_{n+1, i}\right) \prod_{i>j=0}^{n+1} \lambda_{i j}\left(k_{i j}\right) d k_{i j},
\end{aligned}
$$

where

$$
\begin{aligned}
\lambda_{i j}\left(k_{i j}\right)=(2 \pi)^{-4} \int & \exp \left[i k_{i j}\left(y_{i}-y_{j}\right)\right] \\
& \times \exp \left[4 i g^{2} a_{i j} \Delta_{+}\left(y_{i} y_{j}\right)\right] d\left(y_{i}-y_{j}\right)
\end{aligned}
$$

and $a_{i j}=(-1)^{i+j}$ times $1, \frac{1}{2}$ or $\frac{1}{4}$ depending whether none, one, or both $y_{i}$ and $y_{j}$ take on the value 0 or $n+1$. The Fourier variables $k_{i j}$ are defined in the following fashion: Labeling the points $x$ and $x^{\prime}$ in coordinate space by $y_{0}$ and $y_{n+1}$ respectively, the momentum variable $k_{i j}$ is chosen for the $\lambda$ which connects the points $y_{i}$ and $y_{j}$. Thus diagrammatically, there are $n \lambda$ 's "emitted" from the lowest point $x^{\prime}$ "propagating" to the remaining $n$ points. The point $y_{n}$ "reabsorbs" one and in turn emits $n-1$ others, etc.

The problem of giving meaning to the Fourier transformed propagator is essentially, therefore, transferred to that of defining $\lambda(k)$, provided, as will then be the case, the $k$ integrations converge. Since $\lambda(y)$ has as leading singularity near the light cone the form $\exp \left( \pm g^{2} / y_{\mu}{ }^{2}\right)$, we consider first a simple one-dimensional example of the same structure. The function $g(x)=x^{-2} \exp \left(g^{2} / x^{2}\right)$ has the transform

$$
g(k)=\int\left[\exp \left(g^{2} / x^{2}\right)\right] e^{i k x} x^{-2} d x .
$$

Obviously the difficulties in this integration occur for small $x$. It will suffice here, therefore, to discuss the analytic continuation procedure for the quantity ${ }^{13}$

$$
f\left(g^{2}\right)=\int_{0}^{1}\left[\exp \left(g^{2} / x^{2}\right)\right] x^{-2} d x .
$$

In the physically interesting case of $\lambda_{i j}$ when one approaches the light cone from that direction, either space-like ot time-like, such that the exponent approaches $+\infty$, integrals of the type (35) appear.

The function $f\left(-g^{\prime 2}\right)$ exists and is perfectly well behaved. To analytically continue to the region $g^{2}<0$ one notes that

$$
f\left(-g^{\prime 2}\right)=\frac{1}{2}\left(\frac{g^{\prime 2}}{\pi}\right)^{-\frac{1}{2}}-{ }_{1} F_{1}\left(\frac{1}{2}, \frac{3}{2},-g^{\prime 2}\right), \quad g^{\prime 2}>0 .
$$

When $g^{\prime 2}=-g^{2}\left(g^{2}>0\right)$, Eq. (36) has a quite finite analytic continuation. Using the integral representation

${ }^{13}$ The analytic properties of the more general function $g(k)$ has been considered by L. Cooper. 
for ${ }_{1} F_{1}$, one obtains the defining equation for $f\left(g^{2}\right)^{14,15}$ :

$$
f\left(g^{2}\right)=\frac{-i}{2}\left(\frac{g^{2}}{\pi}\right)^{-\frac{1}{2}}-\int_{1}^{\infty}\left[\exp \left(g^{2} / z^{2}\right)\right] z^{-2} d z .
$$

In the physically significant problem, one must Fourier analyze quantities of the type $\lambda_{+}(x)=\exp \left[4 i g^{2} \Delta_{+}(x)\right]$. The exponent may be written as

$$
\begin{aligned}
& 4 i g^{2} \Delta_{+}(x)=i g^{2} / \pi \delta\left(x_{\mu}{ }^{2}\right) \\
& \quad-g^{2} / \pi^{2}\left(x_{\mu}^{2}\right)^{-1}-g^{2} \mu^{2} / 2 \pi^{2} F\left(x^{2}\right),
\end{aligned}
$$

where $F\left(x^{2}\right)$ near the light cone behaves as $\ln \mu|x|$ plus a finite but discontinuous function. To treat the $\delta$ function part we note that

$\exp \left[i g^{2} / \pi \delta\left(x_{\mu}^{2}\right)\right]$

$$
=1+\frac{\delta\left(x_{\mu}^{2}\right)}{\delta(0)}\left\{\exp \left[i g^{2} / \pi \delta(0)\right]-1\right\}=1,
$$

since the second term vanishes everywhere but on the light-cone and is bounded on the cone. Thus the pulse part of the $\Delta_{+}$function does not contribute.

The remaining part of $\lambda(x)$ to be analyzed has as its leading singularity $\left(x_{\mu}^{2}\right)^{-1}$ (which is analogous to our one-dimensional example in the foregoing) and the logarithmic term (which resembles the neutral scalar no-recoil structure treated in 3). The fact that we now have a four dimensional problem manifests itself through the possibility that $1 / x^{2}$ changes sign across the light-cone whereas in one dimension there can be no change of sign. Thus in the Fourier analysis of $\exp \left[-g^{2} / \pi^{2}\left(x_{\mu}^{2}\right)^{-1}\right]$ there is no singularity outside the light-cone while in the regions of $x_{\mu}{ }^{2}$ time-like analytic continuation as in the one-dimensional example defines that portion of the integration in the transform. When $g^{2}$ is large enough, the logarithmic part of $F\left(x^{2}\right)$ will correspond to a branch point in the usual fashion. Thus there will be an analytic continuation required from small $g^{2}$ to large $g^{2}$ even for the regions outside the light cone.

The other type of function whose Fourier analysis must be defined is $\lambda \_(x)=\exp \left[-4 i g^{2} \Delta_{+}(x)\right]$. The only difference in treating this function from the procedure just outlined lies in the interchange of the space-like and time-like regions. Hence all exponentials must be analytically continued, though each in a separate domain.

The statement made above that $\lambda(k)$ will vanish sufficiently rapidly for large $k$ is now seen to hold, since the continuation has ensured that as a function of $x, \lambda$ vanishes adequately at the origin. ${ }^{16}$

\footnotetext{
${ }^{14}$ In obtaining Eq. (37), the usual convention that $(-1)^{\frac{1}{2}}=+i$ has been followed.

15 The fact that the definition of $f\left(g^{2}\right)$ by continuation results in a complex form [Eq. (37)] for the apparently real integral of $\mathrm{Eq}$. (35) may perhaps be a manifestation of a "charge renormalization" akin in this respect to that required in the theory of T. D. Lee [Phys. Rev. 95, 1329 (1954)].

${ }^{16}$ An alternate method of obtaining the finite result of Eq. (37) consists of introducing a "structure" or cutoff function $\phi_{\lambda}(x)$ into
}

It must be remembered that while mathematical meaning has now been given to the theory, the method employed does not necessarily entail that the Fourier transforms so defined behave in momentum space in accordance with physical criteria such as causality and unitarity. These questions require further investigation.

\section{THE EFFECTIVE INTERCCTION SERIES FOR THE RENORMALIZED GREEN'S FUNCTIONS}

The now well-defined series, Eq. (32), aside from being a high-momentum expansion, may also be classified by the number of $\lambda_{i j}$ 's in each term. Since the transformation of the theory to the effective transcendental coupling (as reflected in the structure of the $\lambda$ 's) has presumably regrouped the interaction of the fields into more natural units than the perturbation forms, it appears desirable to re-express the solutions in terms of quantities closely related to the $\lambda$ 's but in addition vanishing when the interaction is turned off. When $g^{2}$ equals zero, $\lambda_{i j}(x)=1$ or $\lambda_{i j}\left(k_{i j}\right)=\delta\left(k_{i j}\right)$. Equation (32) immediately becomes

$$
G_{c}(p)=\sum_{n=0}^{\infty}(-m)^{n}(\gamma p)^{-n-1} \equiv G_{+}{ }^{(0)}(p) .
$$

On the other hand, since $\Delta_{+}(x) \rightarrow 0$ as $x_{\mu} \rightarrow \infty$ (for $x_{\mu}$ not on the light-cone) $\lambda_{i j}(x) \rightarrow 1$ in this limit. Since the light-cone is a hypersurface in the volume integration performed on Fourier analyzing, and since further the analytic continuation makes $\lambda_{i j}(x)$ well behaved on the cone, $\lambda_{i j}\left(k_{i j}\right)$ will have the term $\delta\left(k_{i j}\right)$ arising in a natural fashion. This result could not have been inferred from a perturbation expansion of $\lambda_{i j}$ since the latter is not permissible. Indeed the $\delta\left(k_{i j}\right)$ part of $\lambda_{i j}$ just accounts for the fact that the nucleon has mass, as may be seen from Eq. (40), while the remainder, $\Lambda_{i j}\left(k_{i j}\right)=\lambda_{i j}\left(k_{i j}\right)-\delta\left(k_{i j}\right)$ represents the interaction of the massed nucleon with the meson field. It is in powers of $\Lambda_{i j}$ then, that we shall expand our Green's functions. In the process, the $H_{0}$ 's will be replaced by $G_{+}{ }^{(0)}$ 's as this argument leads one to expect.

To this end, we write the product occurring in Eq. (32) as

$$
\begin{aligned}
\prod_{i>j=1}^{n} \lambda_{i j}\left(k_{i j}\right) & =\prod_{i>j} \delta\left(k_{i j}\right)+\sum_{k>l} \Lambda_{k l}\left(k_{k l}\right) \prod_{i>j} \delta\left(k_{i j}\right) \\
& +\frac{1}{2} \sum_{\substack{k>l \\
s>t}}^{\prime} \Lambda_{k l}\left(k_{k l}\right) \Lambda_{s t}\left(k_{s t}\right) \prod_{i>j}^{\prime \prime}\left(k_{i j}\right)+\cdots
\end{aligned}
$$

where primes on the I's denote omission of indices appearing in the accompanying $\sum$ 's while those on the $\sum$ 's imply that no two $\Lambda$ 's are to have the same indices.

the integrand of $\mathrm{Eq}$. (35). $\phi_{\lambda}(x)$ is taken to have the properties that $\phi_{\lambda} \rightarrow 1$ as $\lambda \rightarrow \infty$ and that convergence of the integral (35) is assured. Equation (37) then results when the transformation $x^{2}=-y^{2}$ (or more precisely $x=i y$ ) is made, followed by rotation of the contour onto the $x$ axis. However, in what follows the continuation viewpoint will be adhered to since no additional physical end is gained by introducing a cut-off function of the type required. 
In Eq. (41) we have considered the product to be only from 1 to $n$. All terms with end points at either $x$ or $x^{\prime}$ were thus neglected. In the $S$-matrix limit when $x, x^{\prime} \rightarrow \pm \infty$ these terms will vanish naturally and hence have been dropped for simplicity.

The first term of Eq. (41) leads to Eq. (40) with the renormalized mass (of course only $m^{\prime}$ appears henceforth). The second term yields

$$
\begin{aligned}
& \begin{aligned}
G_{c}{ }^{(1)}=\sum_{n, \alpha, \beta, \gamma}\left(-m^{\prime}\right)^{n} \int H_{0}{ }^{\alpha}(p) \Lambda_{\beta}(k) \\
\\
\quad \times H_{0}{ }^{\beta}(p-k) H_{0} \gamma(p) d k,
\end{aligned} \\
& \text { where }
\end{aligned}
$$

The structure of Eq. (42) arises from Eq. (32) since for this term there is only one nonzero intermediate momentum, or, diagrammatically, one "emission" or "absorption" which spans $\beta$ of the $H_{0}$ 's. Here and subsequently $\Lambda$ plays the role of the meson propagator. The summation over $\alpha, \beta$ and $\gamma$ extends from 1 to $\infty$ since only internal $\Lambda$ 's are being retained. In order that there be $n+1 H_{0}$ 's, $\alpha, \beta$, and $\gamma$ are restricted by the condition $\alpha+\beta+\gamma=n+1$. The $n$ summation then runs from 2 to $\infty$ so that at least three $H_{0}$ 's occur, one on each edge and one spanned by the $\Lambda$. By straightforward expansion, remembering that $-(p-k)^{-2}=H_{0}^{2}(p-k)$, one easily sees that

$$
\begin{aligned}
& G_{c}^{(1)}(p)=-G_{+}{ }^{0}(p) \\
& \quad \times\left[m^{\prime 2} \int d k \gamma_{5} \frac{m^{\prime} \Lambda_{0}(k)+\gamma(p-k) \Lambda_{1}}{(p-k)^{2}+m^{\prime 2}} \gamma_{5}\right] G_{+}{ }^{(0)}(p) .
\end{aligned}
$$

The $\Lambda_{0}$ and $\Lambda_{1}$ which have opposite signs in their exponents are generalizations of the fact that in $G_{+}{ }^{(0)}(p-k)$ the numerator would have the form $m-\gamma(p-k)$. Indeed a perturbation expansion of the $\Lambda$ 's to lowest order yields the familiar equivalence result between $p s(p s)$ and $p s(p v)$ with $g_{p c}=2 m g$. Of course, such an expansion should not be made and Eq. (44) represents a more general equivalence relation between the two theories when the full interaction unit, $\Lambda(k)$, rather than just the one meson part, is used.

The expansion for $G_{c}$, has been recast into the form

$$
G_{c}=G_{+}{ }^{(0)}-G_{+}{ }^{(0)} \delta M^{(1)} G_{+}{ }^{(0)}+\cdots .
$$

The quantity in the bracket of Eq. (44) may then be associated with an effective first-order "mass operator," orders meaning now powers of $\Lambda$. Every order of this mass operator is, of course, finite now because of analytic continuation and the fact that the explicit infinities have been previously extracted.

The parts quadratic in $\Lambda$ in Eq. (41) give, along with irreducible second-order diagrams, the iterate of $\delta M^{(1)}$. To arrive at the latter, we consider the terms in Eq. (41) where $l>s$ and the symmetric ones where $t>k$.
The portion of $G_{c}{ }^{(2)}$ thus generated has the form

$$
\begin{aligned}
\sum\left(-m^{\prime}\right)^{n} \int H_{0}{ }^{\alpha}(p) H_{0}{ }^{\beta}(p-k) H_{0}{ }^{\gamma}(p) H_{0}{ }^{\delta}\left(p-k^{\prime}\right) \\
\times H_{0}{ }^{\epsilon}(p) \Lambda_{\beta}(k) \Lambda_{\delta}\left(k^{\prime}\right) d k d k^{\prime} .
\end{aligned}
$$

All Greek indices but $\gamma$ sum from 1 to $\infty$, the latter from 0 to $\infty$. It may be verified that (46) reduces to

$$
\begin{aligned}
m^{\prime 4} G_{+}{ }^{(0)}(p) \int d k \gamma_{5} \frac{m^{\prime} \Lambda_{0}(k)+\gamma(p-k) \Lambda_{1}(k)}{(p-k)^{2}+m^{\prime 2}} \\
\times \gamma_{5}\left\{G_{+}{ }^{(0)}(p)+G_{+}{ }^{(0)}(0)\right\} \int d k^{\prime} \gamma_{5} \\
\times \frac{m^{\prime} \Lambda_{0}\left(k^{\prime}\right)+\gamma\left(p-k^{\prime}\right) \Lambda_{1}\left(k^{\prime}\right)}{\left(p-k^{\prime}\right)^{2}+m^{\prime 2}} \gamma_{5} G_{+}{ }^{(0)}(p) .
\end{aligned}
$$

The first term of the brace gives the iterate of $\delta M^{(1)}$. The $G_{+}{ }^{(0)}(0)=1 / m^{\prime}$ term forms a new second order structure characteristic of $p s(p v)$ theory. Diagrammatically, the latter may be viewed as a coalescence of the first term in which the two central vertices coincide. In general, to $n$th order, one will obtain terms corresponding to every diagram of the normal perturbation type with $\Lambda_{0,1}$ playing the role of the meson propagator, plus all diagrams derived from these by allowing any number of interior $G_{+}{ }^{(0)}$ 's to be replaced by $G_{+}{ }^{(0)}(0)$ (as long as no two $\Lambda$ 's coincide). Statements similar to those made for $\delta M^{(1)}$ about equivalence relations hold in these higher terms. ${ }^{17}$

The existence of a mass operator brings with it the possibility of further, but finite, renormalizations. These would have to be evaluated in obtaining physical results, but in principle no difficulty should arise in such a calculation.

Turning to the two-nucleon Green function, $G_{12}{ }^{\prime}\left(x_{1} x_{2}, x_{1}{ }^{\prime} x_{2}^{\prime}\right)$ the functional integral to be performed is now

$$
\begin{aligned}
& G_{12}{ }^{\prime}\left(x_{1} x_{2}, x_{1}{ }^{\prime} x_{2}{ }^{\prime}\right)=N^{-1} \int \delta \phi \\
& \times \exp \left[-\frac{1}{2} i \int \phi(\xi) \Delta_{+}{ }^{-1}\left(\xi \xi^{\prime}\right) \phi\left(\xi^{\prime}\right) d \xi d \xi^{\prime}\right] \\
& \quad \times \sum_{s, t=0}^{\infty}(-m)^{s+t} \int d y_{1} \cdots d y_{n} H_{0}\left(x_{1} y_{1}\right) \cdots H_{0}\left(y_{s} x_{1}{ }^{\prime}\right) \\
& \times \int d z_{1} \cdots d z_{n} H_{0}\left(x_{2} z_{1}\right) \cdots H_{0}\left(z_{t} x_{2}{ }^{\prime}\right) \\
& \quad \times \exp \left[-2 g \gamma_{5}{ }^{1} \int \phi(\xi) f_{1}(\xi) d \xi\right. \\
& \left.\quad-2 g \gamma_{5}{ }^{2} \int \phi(\xi) f_{2}(\xi) d \xi\right]
\end{aligned}
$$

${ }^{17}$ The formulation of the total mass operator in terms of a suitably defined "vertex operator" may be of interest. It must be remembered that the appearance of $\Lambda_{0}$ or $\Lambda_{1}$ depends upon the specific structure to be spanned by the $\Lambda$ 's. 
where $f_{i}(\xi)$ is the series of $\delta$ functions appearing in Eq. (28) and depending parametrically on the $y$ 's and $z$ 's, respectively. Equation (48) may be suitably antisymmetrized at any stage of the calculation, a procedure which need not be considered here. The $\gamma_{5}$ 's of each particle commute with any $\gamma$ 's of the other, of course. One aspect of isotopic spin which may be included if desired, is the use of $\tau_{3}{ }^{i}$ rather than unity to describe the neutral mesons; these matrices may be assumed to have been incorporated into the $\gamma_{5}$ 's. Use of $\tau_{3}$ 's only modifies the $n-p$ forces and would not in any case alter the one-nucleon results. The functional integral is performed in the usual fashion to yield

$$
\begin{gathered}
G_{12}{ }^{\prime}\left(x_{1} x_{2}, x_{1}^{\prime} x_{2}{ }^{\prime}\right)=Z_{2}{ }^{2} \sum_{s, t=0}^{\infty}\left(-m^{\prime}\right)^{s+t} \int d y_{1} \cdots d y_{s} d z_{1} \cdots d z_{t} \\
\times \exp \left[-4 i g^{2} \gamma_{5}{ }^{1} \gamma_{5}{ }^{2} \sum_{i, j=1}^{s, t}(-)^{i+j} \Delta_{+}\left(y_{i}, z_{j}\right)\right] \\
\times \exp \left[4 i g^{2} \sum_{i>j=1}^{s}(-)^{i+j} \Delta_{+}\left(y_{i} y_{j}\right)\right] H_{0}\left(x_{1} y_{1}\right) \cdots H_{0}\left(y_{s} x_{1}{ }^{\prime}\right) \\
\times \exp \left[4 i g^{2} \sum_{i>j=1}^{t}(-)^{i+j} \Delta_{+}\left(z_{i} z_{j}\right)\right] \\
\quad \times H_{0}\left(x_{2} z_{1}\right) \cdots H_{0}\left(z_{t} x_{2}{ }^{\prime}\right)
\end{gathered}
$$

Once again all meson terms leading to either edge of both particles have been omitted. The second and third exponentials are each nucleon's radiative corrections. These will, of course, modify the effects of the first exponential which gives the interaction between the nucleons due to the exchange of mesons. To go over to the more physical form, in terms of $G_{+}{ }^{(0)}$ one again would express each exponential in powers of $\Lambda$. The analog of the basic Mфller interaction can be obtained, however, by dropping all the self-terms and merely expanding the first term:

$$
\begin{gathered}
\exp \left[-4 i g^{2} \gamma_{5}^{1} \gamma_{5}^{2} \sum_{i, j=1}^{s, t}(-)^{i+j} \Delta_{+}\left(y_{i} z_{j}\right)\right] \\
=1+\sum_{i, j} \Lambda_{i j}{ }^{12}\left(y_{i} z_{j}\right)+\cdots \\
\Lambda_{i j}{ }^{12}=\exp \left[-4 i g^{2} \gamma_{5}^{1} \gamma_{5}^{2}(-)^{i+j} \Delta_{+}\left(y_{i} z_{j}\right)\right]-1 .
\end{gathered}
$$

The unity in Eq. (50) leads to the product of the noninteracting Green functions, $G_{+}{ }^{(0)}\left(x_{1} x_{1}{ }^{\prime}\right) G_{+}{ }^{(0)}\left(x_{2} x_{2}{ }^{\prime}\right)$, while the second term produces in momentum space

$$
\begin{aligned}
& G_{c}{ }^{(1)}\left(p_{1} p_{2} k\right)=\sum_{s, t=1}^{\infty}\left(-m^{\prime}\right)^{s+t} \sum_{i, j=1}^{s, t} \Lambda_{i j}{ }^{12}(k) \\
& \quad \times H_{0}{ }^{i}\left(p_{1}\right) H_{0}{ }^{j}\left(p_{2}\right) H_{0}{ }^{s-i+1}\left(p_{1}-k\right) H_{0}{ }^{t-j+1}\left(p_{2}+k\right),
\end{aligned}
$$

where $k$ is the momentum transfer between the nucleons. The $\Lambda_{i j}{ }^{12}(k)$ may be brought past the first two $H_{0}$ factors where it becomes $\Lambda_{00}{ }^{12}$. The sum may then be easily performed to yield

$$
\begin{aligned}
& G_{c}{ }^{(1)}\left(p_{1} p_{2} k\right)=G_{+}{ }^{(0)}\left(p_{1}\right) G_{+}{ }^{(0)}\left(p_{2}\right)\left[m^{\prime 2} \Lambda_{00}{ }^{12}(k)\right] \\
& \times G_{+}{ }^{(0)}\left(p_{1}-k\right) G_{+}{ }^{(0)}\left(p_{2}+k\right),
\end{aligned}
$$

and hence the effective lowest order interaction structure becomes

$$
\begin{gathered}
I_{12}(k)=m^{\prime 2} \Lambda_{00}{ }^{12}(k) \quad \text { or } \\
I_{12}\left(x_{1}-x_{2}\right)=m^{\prime 2}\left\{\exp \left[-4 i g^{2} \gamma_{5}^{1} \gamma_{5}{ }^{2} \Delta_{+}\left(x_{1} x_{2}\right)\right]-1\right\},
\end{gathered}
$$

which displays the usual equivalence relation upon perturbation expansion.

In coordinate space, the $\gamma_{5}$ dependence of $I_{12}$ can be brought out of the exponent as $\left(i \gamma_{5}^{1} \gamma_{5}^{2}\right)^{2}=-1$ :

$$
\begin{aligned}
I_{12}\left(x_{1}-x_{2}\right)=m^{\prime 2}\left[\left\{\cos 4 g^{2} \Delta_{+}\left(x_{1} x_{2}\right)-1\right\}\right. \\
\\
\left.-i \gamma_{5}^{1} \gamma_{5}^{2} \sin 4 g^{2} \Delta_{+}\left(x_{1} x_{2}\right)\right] .
\end{aligned}
$$

This interaction is, of course, complex, the imaginary part being related to the elastic scattering potential while the real part corresponds to decay out of the two nucleon state, that is to meson and pair production. The adiabatic potential is the time integral of the imaginary part of $I_{12}$. A preliminary analysis indicates that for $p-p$ forces the central potential has a repulsive core. Of course, the validity of an adiabatic approximation is questionable in a theory involving $\gamma_{5}$ 's, ${ }^{18}$ and more detailed analysis is required to obtain a meaningful result.

The meson-nucleon Green's function may be obtained either by straightforward functional integration by way of Eq. (4) or more easily from the expression

$$
\begin{array}{r}
G_{M N}^{\prime}\left(x \eta, x^{\prime} \eta^{\prime}\right)=2 \int \Delta_{+}(\eta \xi)\left[\frac{\delta}{\Delta_{+}\left(\xi \xi^{\prime}\right)} G_{+}^{\prime}\left(x x^{\prime}\right)\right] \\
\times \Delta_{+}\left(\xi^{\prime} \eta^{\prime}\right) d \xi d \xi^{\prime}
\end{array}
$$

One can thus get directly the result to first order in $\Lambda$ from Eq. $(44)^{19}$ :

$$
\begin{aligned}
& G_{c}^{(1)}\left(x \eta, x^{\prime} \eta^{\prime}\right)=\int \Delta_{+}(\eta \xi) G_{+}{ }^{(0)}(x-\xi) \\
& \times\left\{-4 i g^{2} m^{\prime 2} \gamma_{5}\left[m^{\prime} \lambda_{0}\left(\xi-\xi^{\prime}\right) \Delta_{m^{\prime}}\left(\xi \xi^{\prime}\right)\right.\right. \\
& \left.\left.-\lambda_{1}\left(\xi-\xi^{\prime}\right) \gamma p_{\xi} \Delta_{m^{\prime}}\left(\xi \xi^{\prime}\right)\right] \gamma_{5}\right\} \\
& \quad \times G_{+}{ }^{(0)}\left(\xi^{\prime} x^{\prime}\right) \Delta_{+}\left(\xi^{\prime} \eta^{\prime}\right) d \xi d \xi^{\prime},
\end{aligned}
$$

where $\Delta_{m^{\prime}}$ is $\Delta_{+}$with mass $m^{\prime}$. When $\lambda_{0,1}$ is replaced by unity, one again recognizes the characteristic equivalence with $p s(p s)$ theory, the process of differentiation having replaced $\Lambda$ by $\lambda$ in the interaction structure. The approximation of Eq. (56) may be of use for lowenergy scattering since a single $\lambda$ already involves many virtual mesons. ${ }^{20}$

${ }^{18}$ R. Arnowitt and S. Gasiorowicz, Phys. Rev. 94, 1057 (1954).

${ }_{19}$ We have omitted differentiations of $m^{\prime}$ and $Z_{2}$ which would lead to terms with two meson vertices coinciding.

20 The behavior of the theory at low energy can also be inferred from threshold theorems. These may be derived by inserting an external meson field into the Lagrangian. A constant pseudoscalar field produces no effects, indicating that, aside from $\mu / m$ corrections, the cross section goes to zero at threshold. To arrive at the $p$-shift one must insert a field $g \phi(x)=\alpha_{\mu} x_{\mu}$ where $\alpha_{\mu}$ is a constant vector. The vertex can then be generated by the operation $\Gamma(p, p+k) \cong k_{\mu} \partial / \partial \alpha_{\mu} G^{-1}(p, \alpha)$. 
Production processes in both meson-nucleon and nucleon-nucleon collisions can be calculated from the real part of the respective interaction structures. The exponential dependence upon the meson field in these terms should imply that multiple meson production is favored. Such questions have been discussed by Glauber $^{11}$ in the semiclassical approximation which is closely related to the first term in our expansion in $\Lambda$. The conclusions there seem to support such a conjecture.

\section{CONCLUSION}

In the early examples of this paper a number of more or less severe approximations were made in order to obtain closed form solutions. The aim in so doing was to exhibit the salient differences between "renormalizable" and "unrenormalizable" theories, rather than to obtain physical predictions at this juncture. Although the examples discussed varied considerably in aspect, the fundamental derivative nature of the coupling produced the striking essential singularity behavior both of the renormalization constants and of the renormalized kernels. When one uses a nonperturbation approach to even the renormalizable theories (such as neutral scalar), another phenomenon arises in that a critical coupling constant exists above which analytic continuation must be performed if the transformation theory is to be maintained. Rigorously, in the gradient theories, the essential singularity causes the critical coupling constant to be zero.

The perturbation renormalization procedure would seem, in view of the above, to require of the theories to which it is applied a certain structure such that though an expansion near the origin (i.e., around a branch point) is not permitted, meaning can still be given to the individual terms. Even if the theory is mild enough so that such a technique is feasible, the perturbation approach would still not tell one of the existence of the critical coupling value and the need for analytic continuation. In the unrenormalizable case the situation is more serious still. Assuming one had been able to extract the explicitly infinite terms order by order (via some prior knowledge of the exact results) the ostensibly renormalized perturbation expansion would still be divergent in a really intractable manner, as analytic continuation, of its very nature, can only give meaning to the closed form expression.

In the beta-decay example treated in the appendix the difficulties encountered in attempting to render the theory finite are of a different nature since here one is dealing with something resembling a vacuum polarization effect. Insofar as one can learn from the perturbation expansion (to which our own criticisms above may apply) the renormalization in the $S$-matrix limit seems perhaps possible, unlike the finite-times Green's functions. There is perhaps an alternate way of treating the problem. In view of the equivalence between a sufficiently heavy intermediate meson field and a direct interaction, the beta coupling could have been discussed as the limiting case of normal meson couplings. Since now a number of these may be renormalized, then at least in the case of such couplings, the problem is reduced to the consideration of whether the infinite meson mass limit leaves the renormalized structure finite.

The specific approximations which have been made in Secs. 3 and 4 were two in number. The first, the no recoil assumption, reduced the partial differential equation for $G_{+}(\phi)$ to an ordinary first-order equation. ${ }^{21}$ The second approximation used consists in neglecting the commutator of the various Dirac matrices. The use of these assumptions cannot only change the numerical validity of the results (which would not concern us here) but in some cases can destroy the basic requirements that these results must obey. An example of this was seen in Sec. 4. Such questions are perhaps most significant in theories involving $\gamma_{5}$ since this matrix does not have an unambiguous nonrelativistic reduction. For these reasons, it is only insofar as the above results agree with the full recoil theory that they are trustworthy. For the relativistic case, however, it was seen that the infinities are again of the essential singularity type. These can then be removed in the physically acceptable way of the renormalization philosophy. Within the framework of our solution, these strong divergences imply that the remainder after this first renormalization must have equally virulent behavior in the zero time limit of the kernels since the canonical commutation relations are preserved. Hence a further definition is necessary at this point, a process which in "renormalizable" theories is required only in certain domains of the coupling constant (above a critical size) and, hence, there for a much milder problem. As in other examples of redefinition of a theory, it seems reasonable to judge the procedure by the physical usefulness of the results; in particular, the fundamental requirements on the analytic behavior in momentum space must be maintained.

The approximate forms specifically exhibited for several physically important interaction operators of the theory seem promising in those aspects where $p s(p v)$ has always been thought to be of interest, such as multiple meson production and nuclear forces. However, before any predictions (even rigorous ones) can be taken as more than indicative, it should be remembered that several important qualifications apply. The most physical of these has been the neglect of the charged mesons. Had the latter been included none of the steps of this method could have been performed, and in particular no definite statement of renormalizability been made. ${ }^{22}$ Analogies from perturbation theory here

\footnotetext{
${ }^{21}$ When recoil is dropped, vacuum polarization effects automatically vanish, of course.

${ }_{22}$ Inclusion of charged mesons presents many of the same problems as are encountered in treating the spin if one does not make the assumption of commutativity. Such an approximation may correspond to a weak coupling one and not be as serious as in
} 
do not seem of much aid. At the moment then, charge symmetry can only be discussed in an ad hoc fashion. The other major omission is concerned with closed loop effects. The problem here is again one of the continued applicability of the renormalization approach. Since the only methods known at present to deal with vacuum polarization are at least of a quasi-perturbation nature, no conclusions can be drawn.

\section{ACKNOWLEDGMENTS}

We wish"to thank Dr. J. R. Oppenheimer for several interesting "conversations and for the hospitality extended us at The Institute for Advanced Study.

\section{APPENDIX A. THE FERMI INTERACTION IN THE STATIC LIMIT}

A second class of "unrenormalizable" theories consists of the beta interactions. We consider here the nucleon static limit for the scalar coupling. ${ }^{23}$ The Lagrangian for such a system may be written in a unified notation as

$$
\begin{aligned}
\mathscr{L}=-\frac{1}{2} \bar{\psi}\left(-p_{0}+m\right) \psi-\frac{1}{2} \bar{\phi}\left(\gamma p+\lambda m_{e}\right) & \\
& -g \bar{\psi} \alpha_{1} \psi \bar{\phi} \alpha_{2} \phi+\bar{\eta} \phi+\text { herm conj, }
\end{aligned}
$$

where $\psi$ represents the nucleon field, $\phi$ the lepton field (which is, of course, kept relativistic),

$$
\psi=\left(\begin{array}{l}
\psi_{p} \\
\psi_{n}
\end{array}\right), \quad \phi=\left(\begin{array}{l}
\phi_{e} \\
\phi_{\nu}
\end{array}\right)
$$

$\lambda$ is a lepton mass matrix (unity when acting on electron functions, zero when on neutrino functions) and $\alpha_{1}$ and $\alpha_{2}$ are the matrices [in the spaces defined by Eq. (A2)] required to allow the appropriate processes to take place. For simplicity, however, we shall in what follows set $\lambda$, $\alpha_{1}$ and $\alpha_{2}$ to unity. $\eta$ and $\bar{\eta}$ are external spinor sources which are assumed to anticommute with all other fermion amplitudes. By use of these external sources, the functional differential equation for the one-nucleon Green's function may be obtained in a fashion analogous to the case in which the $\phi$ field is a boson, though slightly more care must be taken due to the anticommutativity of the fermion sources:

$$
\begin{array}{r}
{\left[-p_{0}+m-g\left\{\frac{1}{i} \frac{\delta_{l}}{\delta \bar{\eta}(t)}+\phi(t)\right\}\left\{\frac{1}{i} \frac{\delta_{r}}{\delta \eta(t)}+\bar{\phi}(t)\right\}\right]} \\
\times G_{+}{ }^{\prime}\left(t, t^{\prime} ; \bar{\phi} \phi\right)=\delta\left(t-t^{\prime}\right) .
\end{array}
$$

Here subscripts " $l$ " and " $r$ " mean left and right derivatives respectively, that is, in performing the functional differentiations indicated, one takes the variation with respect to the source, and commutes it to the

\footnotetext{
the spin reduction since there is no question of a $\gamma_{5}$-like fourth component in this three-dimensional isotopic space.

${ }^{23}$ Such an approximation is actually valid for allowed beta transitions with this coupling.
}

extreme left or right in the expression before dividing by it.

From Eq. (A3) one may again follow the usual procedure $^{3,4}$ to arrive at the result

$$
\begin{array}{r}
G_{+}{ }^{\prime}\left(t-t^{\prime}\right)=N^{-1} \int \exp \left[-i \int \phi(\xi) G_{0}^{-1}\left(\xi-\xi^{\prime}\right) \phi\left(\xi^{\prime}\right)\right] \\
\times G_{+}\left(t, t^{\prime} ; \bar{\phi} \phi\right) \delta \bar{\phi} \delta \phi,
\end{array}
$$

where $\left(\gamma p+m_{e}\right) G_{0}=1$ and

$$
\left[-p_{0}+m+g \bar{\phi}(t) \phi(t)\right] G_{+}\left(t, t^{\prime} ; \bar{\phi} \phi\right)=\delta\left(t-t^{\prime}\right) .
$$

In the functional integral of Eq. (A4) it must be remembered that the $\phi$ 's and $\delta \phi$ 's still anticommute among themselves. ${ }^{24}$ Equation (A5) may be integrated to yield the familiar exponential form but bilinear in the lepton variables. The functional integration may still be performed, however, since the integrand is just a Gaussian with a modified propagator:

$$
\begin{aligned}
G_{+}{ }^{\prime}\left(t-t^{\prime}\right)=i \theta\left(t-t^{\prime}\right) e^{-i m\left(t-t^{\prime}\right)} & \\
& \times \exp \left[-\operatorname{Tr} \ln \left(1+g P G_{0}\right)\right],
\end{aligned}
$$

where in coordinate space $P\left(\xi, \xi^{\prime}\right)=H\left(\xi_{0}, t t^{\prime}\right) \delta^{3}(\xi) \delta^{4}$ $\times\left(\xi-\xi^{\prime}\right)$. The $\operatorname{Tr}$ above refers to a trace both over the spinor indices and coordinates of the leptons. The nunucleon variables, $t$ and $t^{\prime}$, still enter parametrically in $P\left(\xi, \xi^{\prime}\right)$. Structures similar to the above appear in the usual vacuum polarization effects. This is not surprising since one is here dealing with a spinor pair theory coupled to a heavy source, which may be likened to vacuum polarization in an external field.

The logarithm in Eq. (A6) may be expanded in a perturbation series in $g$ to yield

$$
\begin{aligned}
& -\operatorname{Tr} \ln \left(1+g P G_{0}\right)=-\int d \xi \\
& \quad \times\left(\xi\left|g P G_{0}-\frac{1}{2} g^{2}\left(P G_{0}\right)^{2}+g^{3} / 3\left(P G_{0}\right)^{3}-\cdots\right| \xi\right) .
\end{aligned}
$$

If $\xi$ is a representation which diagonalizes the operator $P G_{0}$, then Eq. (A7) may easily be rewritten $a^{25}$

$$
-\operatorname{Tr} \ln \left(1+g P G_{0}\right)=-\delta(0) \int d \xi \ln [1+g f(\xi)]
$$

where $\left(\xi\left|P G_{0}\right| \xi^{\prime}\right)=f(\xi) \delta\left(\xi-\xi^{\prime}\right)$. While to find the diagonalizing representation in general would be very difficult, when $t-t^{\prime} \equiv T$ is very large (the $S$-matrix limit), $P G_{0}$ is diagonal in the $p_{0}$ part of the usual momentum representation. For this partial diagonalization,

${ }^{24}$ For a detailed discussion of functional integrals over fermion variables see P. T. Matthews and $\cdot A$. Salam (to be published).

${ }_{25}$ If, for example, $\xi$ were momentum variables, then $\delta(0)$ is to be regarded as $V T$. In our case, the $\delta(0)$ actually cancels at finite times in the term by term intregation of the perturbation series. 
an equation similar to (A8) can be written

$$
\begin{aligned}
-\operatorname{Tr} \ln \left(1+g P G_{0}\right)=-i T \int & d p_{0} / i T r^{\prime} \\
& \times \ln \left[\left(1+g P G_{0}\left(p_{0}\right)\right],\right.
\end{aligned}
$$

where $\operatorname{Tr}^{\prime}$ is the trace over all remaining eigenvalues. The integral in Eq. (A9) is thus the mass renormalization (or zero-point energy in fixed source language). In the large time limit, therefore, the Green's function reduces to the unperturbed one with renormalized mass and $Z_{2}$ (the latter not appearing in the above calculation). The lepton-nucleon Green function in the same limit exhibits, in addition to these, a coupling constant renormalization.

When the times are not infinite, these simplifications are lost and one must have recourse to the perturbation expansion of Eq. (A7). To first order in $g$ one obtains $-i\left(t-t^{\prime}\right) 4 m g(-i) \Delta_{+}(0)$ which is a mass renormalization. The second term displays both $Z_{2}$ and mass renormalization as well as a finite function of $\left(t-t^{\prime}\right)$ (which vanishes at infinity). However, the $g^{3}$ term involves, aside from finite terms and a $Z_{2}$ renormalization, the product of infinite terms and finite functions of time (which also go to zero at infinity). These presumably would represent $g$ renormalizations but there appear to be terms beyond those necessary to renormalize the second order structure. ${ }^{26}$ Thus to renormalize the theory for finite times it may be necessary to obtain a nonperturbation solution or perhaps to introduce other counter terms. However, even within the $S$-matrix renormalization program, it should be checked whether a unique charge renormalization suffices there in treating all the higher Green functions.

\section{APPENDIX B. STATIC APPROXIMATION WITH GRADIENT COUPLING}

A more complex approximation to the full $p s(p v)$ theory than the example of Sec. 3 involves the gradient part of the coupling term:

$$
\left(-p_{0}+m-i g \sigma \cdot \nabla \phi\right) G_{+}\left(t, t^{\prime} ; \phi\right)=\delta\left(t-t^{\prime}\right),
$$

where $\sigma_{k}=\gamma_{5} \gamma_{0} \gamma_{k}$. Equation (B1) can be formally integrated in terms of time-ordered exponentials. However, the evaluation of such a structure in closed form is, of course, an insoluble mathematical problem. We consider here only the simplified approximation in which the $\sigma$ 's are taken to commute. ${ }^{27}$ With this assumption the ordered exponentials are equivalent to ordinary

${ }^{26}$ For the scalar pair theory, whose charge renormalization has been treated by A. Klein and B. McCormick [Phys. Rev. 98, 1428 (1955)] in the $S$-matrix limit, one obtains a linear and logarithmic divergence at finite times. The linear divergence correctly renormalizes the $g^{2}$ term, while the logarithm fails to do so.

27 This might be viewed as a semiclassical approximation to the cutoff theory considered by G. Chew, Phys. Rev. 94, 1749 (1954). ones, allowing the functional integral to be performed.

$$
\begin{aligned}
G_{+}{ }^{\prime}\left(t-t^{\prime}\right) & =i \theta\left(t-t^{\prime}\right) \exp \left[\left(-i m\left(t-t^{\prime}\right)\right]\right. \\
\times \exp & {\left[-\frac{1}{2} i g^{2} \int_{t^{\prime}}^{t} \int_{t^{\prime}}^{t} d \tau d \tau^{\prime} \nabla^{2} \Delta_{+}\left(0, \tau-\tau^{\prime}\right)\right] . }
\end{aligned}
$$

The exponential is similar to the result of neutral scalar theory except for the Laplacian. The latter raises the order of infinity of the renormalization constants to be extracted. By use of the equation obeyed by $\Delta_{+}, G_{+}{ }^{\prime}$ may be rewritten as

$$
\begin{aligned}
& G_{+}{ }^{\prime}\left(t-t^{\prime}\right)=i \theta\left(t-t^{\prime}\right) e^{-i m\left(t-t^{\prime}\right)} \\
& \times \exp \left[-i g^{2}\left\{\Delta_{+}\left(t-t^{\prime}\right)-\Delta_{+}(0)\right\}-\frac{1}{2} i g^{2} \mu^{2} \int_{t^{\prime}}^{t} \int_{t^{\prime}}^{t} d \tau d \tau^{\prime}\right. \\
&\left.\times \Delta_{+}\left(\tau-\tau^{\prime}\right)+\frac{1}{2} i g^{2} \delta^{3}(0)\left(t-t^{\prime}\right)\right] .
\end{aligned}
$$

Again essential singularities of the type encountered in the $\gamma_{5} \partial_{0} \phi$ approximation arise through the first part of the exponent. The second term is equivalent to the no-recoil scalar theory [with $(i g)^{2} \mu^{2}$ playing the role of $\left.g_{s}{ }^{2}\right]$ which again is equal to the adiabatic limit of the $p s(p s)$ case. $^{4}$ This will produce a further $Z_{2}$ as well as a mass renormalization. The third term is the usual contact structure that occurs in $p s(p s)-p s(p v)$ equivalence transformations and is absorbed into the mass term. Physical effects will arise in the higher Green functions from the scalar part of the interaction.

Interactions involving higher powers of the gradient $\left(\Gamma \phi \sim \nabla^{2 n} \phi\right)$ can similarly be handled.

\section{APPENDIX C. FORMAL EQUIVALENCE OF THE SOLUTION WITH PERTURBATION THEORY}

In this appendix we show that the solution given in Eq. (29) is formally equivalent to the usual perturbation expansion. It will suffice to consider here only the terms of order $g^{2}$ Equation (29), when expanded gives

$$
\begin{aligned}
& G_{g^{2^{\prime}}}\left(x x^{\prime}\right)=i g^{2} \sum_{n=0}^{\infty}(-m)^{n} \int d y_{1} \cdots d y_{n} H_{0}\left(x y_{1}\right) \cdots \\
& \times H_{0}\left(y_{n} x^{\prime}\right)\left[(1+2 n) \Delta_{+}(0)-(-)^{n} \Delta_{+}\left(x x^{\prime}\right)\right. \\
& +2 \sum_{i=1}^{n}(-)^{i}\left[\Delta_{+}\left(x y_{i}\right)+(-)^{n+1} \Delta_{+}\left(y_{i} x^{\prime}\right)\right] \\
& \left.+4 \sum_{i>j=1}^{n}(-)^{i+i} \Delta_{+}\left(y_{i} y_{j}\right)\right] .
\end{aligned}
$$

This is to be compared with

$$
\begin{aligned}
& -\left(x\left|G_{+}{ }^{(0)} \delta M_{g^{2}} G_{+}{ }^{(0)}\right| x^{\prime}\right)=-i g^{2} \int d y d y^{\prime} G_{+}{ }^{(0)}(x y) \\
& \times \gamma_{5} \gamma_{\mu} G_{+}{ }^{(0)}\left(y y^{\prime}\right) \gamma_{5} \gamma_{\nu}\left[i \partial_{\mu} i \partial_{\nu}{ }^{\prime} \Delta_{+}\left(y y^{\prime}\right)\right] G_{+}{ }^{(0)}\left(y^{\prime} x^{\prime}\right) .
\end{aligned}
$$


The two derivatives on $\Delta_{+}\left(y y^{\prime}\right)$ due to the vertices are integrated by parts to act on the appropriate $G_{+}{ }^{(0)}$ 's. Using the defining equation for $G_{+}{ }^{(0)}$ one then obtains

$$
\begin{gathered}
i g^{2}\left[\Delta_{+}(0)\left\{G_{+}{ }^{(0)}\left(x x^{\prime}\right)-2 m \int d y G_{+}{ }^{(0)}(x y) G_{+}{ }^{(0)}\left(y x^{\prime}\right)\right\}\right. \\
-\Delta_{+}\left(x x^{\prime}\right) \gamma_{5} G_{+}{ }^{(0)}\left(x x^{\prime}\right) \gamma_{5} \\
+2 m \int d y \Delta_{+}(x y) \gamma_{5} G_{+}{ }^{(0)}(x y) \gamma_{5} G_{+}{ }^{(0)}\left(y x^{\prime}\right) \\
+2 m \int d y \gamma_{5} G_{+}{ }^{(0)}(x y) \gamma_{5} G_{+}{ }^{(0)}\left(y x^{\prime}\right) \Delta_{+}\left(y x^{\prime}\right) \\
-4 m^{2} \int d y d y^{\prime} \Delta_{+}\left(y y^{\prime}\right) G_{+}{ }^{(0)}(x y) \\
\left.\times \gamma_{5} G_{+}{ }^{(0)}\left(y y^{\prime}\right) \gamma_{5} G_{+}{ }^{(0)}\left(y^{\prime} x^{\prime}\right)\right]
\end{gathered}
$$

In the derivation of Eq. (C3) a term having as coefficient $\left.\partial_{\mu} \Delta_{+}(y)\right]_{y=0}$ has been dropped since this factor vanishes by oddness. Equation (C3) need only be expanded in a power series in $m$ to prove it equivalent to $(\mathrm{C} 1)$.

The terms in which either of the arguments of $\Delta_{+}$is $x$ or $x^{\prime}$ vanish in $S$-matrix limit. The last term is the usual $p s(p s)$ lowest order correction. The coefficient of the remaining $G_{+}{ }^{(0)}\left(x x^{\prime}\right)$ is recognizable even in the perturbation expansion as a $Z_{2}$ renormalization while the other $\Delta_{+}(0)$ term is similarly a mass renormalization. The $p s(p s)$ structure produces its own infinities. However in the rigorous form terms of this sort add back in such a way that the $\Delta_{+}\left(y y^{\prime}\right)$ is replaced by $\exp \left[ \pm 4 i g^{2} \Delta_{+}\left(y y^{\prime}\right)\right]$. When analytic continuation is employed, what remains is finite, which is necessary since higher powers of $\Delta_{+}\left(y y^{\prime}\right)$ appear in subsequent orders and would not be renormalizable.

It may be noted that the formal equivalence with perturbation theory is demonstrable for higher Green's functions as well. However, as was pointed out in Eq. (39), the $\delta\left(x_{\mu}^{2}\right)$ part of $\Delta_{+}(x)$ does not contribute to $\exp \left[4 i g^{2} \Delta_{+}(x)\right]$ but would produce effects in the expanded forms.

\section{APPENDIX D. NEUTRAL $S(V)$ THEORY}

The relativistic counterpart of the nonrecoil case with $\partial_{0} \phi$ coupling is, of course, the $s(v)$ theory which will be considered here. The equation for $G_{+}(\phi)$ is

$$
\left(\gamma p+m+g \gamma_{\mu} \partial_{\mu} \phi\right) G_{+}\left(x, x^{\prime} ; \phi\right)=\delta\left(x-x^{\prime}\right) .
$$

Letting $G_{+}\left(x, x^{\prime} ; \phi\right)=e^{-i g \phi(x)} H_{+}\left(x x^{\prime} ; \phi\right) e^{+i g \phi\left(x^{\prime}\right)}$ one easily finds that

$$
(\gamma p+m) H_{+}\left(x, x^{\prime} ; \phi\right)=\delta\left(x-x^{\prime}\right)
$$

or $H_{+}\left(x, x^{\prime}\right)=G_{+}{ }^{(0)}\left(x, x^{\prime}\right)$. The functional integral is trivial to perform here, yielding

$$
G_{+}{ }^{\prime}\left(x, x^{\prime}\right)=Z_{2} \exp \left[-i g^{2} \Delta_{+}\left(x, x^{\prime}\right)\right] G_{+}{ }^{(0)}\left(x, x^{\prime}\right),
$$

where $Z_{2}=\exp \left[i g^{2} \Delta_{+}(0)\right]$. The structure indicates the well-known fact that the $s(v)$ theory has no physical effects.

$Z_{2}$ in this case is less than unity according to the interpretation given to $\Delta_{+}(0)$ in Sec. 5. In order to give meaning to $G_{c}(p)$, however, analytic continuation is still required just as in the $p s(p v)$ theory. 